\title{
ON THE RATIO OF PEARSON TYPE VII AND BESSEL RANDOM VARIABLES
}

\author{
SARALEES NADARAJAH AND SAMUEL KOTZ
}

Received 26 October 2004

The exact distribution of the ratio $|X / Y|$ is derived when $X$ and $Y$ are, respectively, Pearson type VII and Bessel function random variables distributed independently of each other. The work is motivated by previously published approximate relationships between these two distributions. An application of the result is provided by computing "correction factors" for some of these approximations.

\section{Introduction}

For given random variables $X$ and $Y$, the distribution of the ratio $X / Y$ is of interest in biological and physical sciences, econometrics, and ranking and selection. Examples include Mendelian inheritance ratios in genetics, mass to energy ratios in nuclear physics, target to control precipitation in meteorology, and inventory ratios in economics. Another important example is the stress-strength model in the context of reliability. It describes the life of a component which has a random strength $Y$ and is subjected to random stress $X$. The component fails at the instant that the stress applied to it exceeds the strength and the component will function satisfactorily whenever $Y>X$. Thus, $\operatorname{Pr}(X<Y)$ is a measure of a component reliability. It has many applications especially in engineering concepts such as structures, deterioration of rocket motors, static fatigue of ceramic components, fatigue failure of aircraft structures and aging of concrete pressure vessels.

The distribution of $X / Y$ has been studied by several authors especially when $X$ and $Y$ are independent random variables and come from the same family. For instance, see Marsaglia [14] and Korhonen and Narula [9] for normal family, Press [18] for Student's $t$ family, Basu and Lochner [1] for Weibull family, Shcolnick [23] for stable family, Hawkins and Han [5] for non-central chi-squared family, Provost [19] for gamma family, and Pham-Gia [17] for beta family. However, there is relatively little work of this kind when $X$ and $Y$ belong to different families. In the applications mentioned above, it is indeed quite possible that $X$ and $Y$ could arise from different but similar distributions.

Pearson type VII distributions (which contain Student's $t$ distributions as particular cases) are becoming of increasing importance in classical as well as in Bayesian statistical 
modeling. These distributions have been perhaps unjustly overshadowed-for at least seventy years-by the normal distribution. Pearson type VII distributions are of central importance in statistical inference. Their applications are a very promising path to take. Classical analysis is soundly bend on the normal distribution while Pearson type VII distributions (in particular, Student's $t$ distributions) offer a more viable alternative with respect to real-world data particularly because its tails are more realistic. We have seen unexpected applications in novel areas such as cluster analysis, discriminant analysis, multiple regression, robust projection indices and missing data imputation.

Pearson type VII distributions (in particular, Student's $t$ distributions) for the past fifty years have also played a crucial role in Bayesian analysis. They serve as the most popular prior distribution (because elicitation of prior information in various physical, engineering and financial phenomena is closely associated with Student's $t$ distributions) and generate meaningful posterior distributions. For further discussion of applications, the reader is referred to Kotz and Nadarajah [11].

On the other hand, Bessel function distributions (which contain logistic distributions as particular cases) have found applications in a variety of areas that range from image and speech recognition and ocean engineering to finance. They are rapidly becoming distributions of first choice whenever "something" with heavier than Gaussian tails is observed in the data. For further discussion of applications, the reader is referred to Kotz et al. [10].

There has been considerable work on the relationship between the Student's $t$ and logistic distributions (which are particular cases of Pearson type VII and Bessel function distributions, resp.). The similarities in the shapes of the logistic and the normal distributions have been noted by several authors. An excellent summary of these properties is found in Johnson and Kotz [7]. However, Mudholkar and George [15] showed that the Student's $t$ distribution function with 9 degrees of freedom, when standardized to have variance one provides a better fit of a standardized logistic distribution than the standard normal. George and Ojo [3] and George et al. [2] extended this result by showing that

$$
\operatorname{Pr}(T \leq t) \approx \frac{1}{1+\exp (-a t)}
$$

where $T$ is a Student's $t$ random variable with $\nu$ degrees of freedom and $a=\pi \sqrt{(\nu-2) /(3 \nu)}$. This approximation (1.1) was obtained by equating the cumulant of the Student's $t$ distribution with that of the logistic distribution. The approximation was found to be accurate to two decimal places for middle values of $t$ and to three decimal places at the tails. A better approximation between the Student's $t$ and logistic distributions has been recently proposed by Li and De Moor [13].

The above discussion naturally raises the important question: what is the exact distribution of the ratio of Student's $t$ and logistic random variables? This question does not appear to have been addressed in the literature. In this paper, we discuss the more general problem: the exact distribution of the ratio $|X / Y|$ when $X$ and $Y$ are independent random 
variables having the Pearson type VII and Bessel function distributions with the pdfs

$$
\begin{gathered}
f(x)=\frac{\Gamma(M-1 / 2)}{\sqrt{N \pi} \Gamma(M-1)}\left(1+\frac{x^{2}}{N}\right)^{1 / 2-M}, \\
f(y)=\frac{|y|^{m}}{\sqrt{\pi} 2^{m} b^{m+1} \Gamma(m+1 / 2)} K_{m}\left(\left|\frac{y}{b}\right|\right),
\end{gathered}
$$

respectively, for $-\infty<x<\infty,-\infty<y<\infty, b>0, m>1, M>1$ and $N>0$, where

$$
K_{m}(x)=\frac{\sqrt{\pi} x^{m}}{2^{m} \Gamma(m+1 / 2)} \int_{1}^{\infty}\left(t^{2}-1\right)^{m-1 / 2} \exp (-x t) d t
$$

is the modified Bessel function of the second kind. We also provide an application section and compute "correction factors" for the approximation provided by (1.1).

The Pearson type VII distribution is related to the Student's $t$ distribution as follows: if $M=1+a / 2$ and

$$
U=\sqrt{\frac{a}{N}} X
$$

then $U$ is a Student's $t$ random variable with degrees of freedom $a$. Note that the pdf of a Student's $t$ random variable with degrees of freedom $v$ is given by

$$
f(x)=\frac{1}{\sqrt{\nu} B(\nu / 2,1 / 2)}\left(1+\frac{x^{2}}{\nu}\right)^{-(1+\nu) / 2}
$$

for $-\infty<x<\infty$. Nadarajah and Kotz [16] have shown that the cdf corresponding to (1.6) can be expressed as

$$
F(x)= \begin{cases}\frac{1}{2}+\frac{1}{\pi} \arctan \left(\frac{x}{\sqrt{\nu}}\right)+\frac{1}{2 \pi} \sum_{l=1}^{(\nu-1) / 2} B\left(l, \frac{1}{2}\right) \frac{v^{l-1 / 2} x}{\left(\nu+x^{2}\right)^{l}}, & \text { if } \nu \text { is odd, } \\ \frac{1}{2}+\frac{1}{2 \pi} \sum_{l=1}^{\nu / 2} B\left(l-\frac{1}{2}, \frac{1}{2}\right) \frac{v^{l-1} x}{\left(\nu+x^{2}\right)^{l-1 / 2}}, & \text { if } \nu \text { is even. }\end{cases}
$$

The representations in (1.5) and (1.7) will be crucial for the calculations of this note. The calculations involve the Euler psi function defined by

$$
\Psi(x)=\frac{d \log \Gamma(x)}{d x}
$$


the Struve function defined by

$$
H_{\nu}(x)=\frac{2 x^{\nu+1}}{\sqrt{\pi} 2^{\nu+1} \Gamma(\nu+3 / 2)} \sum_{k=0}^{\infty} \frac{1}{(3 / 2)_{k}(\nu+3 / 2)_{k}}\left(-\frac{x^{2}}{4}\right)^{k},
$$

the Bessel function of the first kind defined by

$$
J_{\nu}(x)=\frac{x^{\nu}}{2^{\nu} \Gamma(\nu+1)} \sum_{k=0}^{\infty} \frac{1}{(\nu+1)_{k} k !}\left(-\frac{x^{2}}{4}\right)^{k}
$$

the hypergeometric functions defined by

$$
E(a ; x)=\sum_{k=0}^{\infty} \frac{1}{(a)_{k}} \frac{x^{k}}{k !}, \quad H(a ; b, c ; x)=\sum_{k=0}^{\infty} \frac{(a)_{k}}{(b)_{k}(c)_{k}} \frac{x^{k}}{k !}
$$

and, the Lommel functions defined by

$$
\begin{aligned}
s_{\mu, \nu}(x)= & \frac{x^{\mu+1}}{(\mu-\nu+1)(\mu+\nu+1)} H\left(1 ; \frac{\mu-\nu+3}{2}, \frac{\mu+\nu+3}{2} ;-\frac{x^{2}}{4}\right), \\
S_{\mu, \nu}(x)= & s_{\mu, \nu}(x)+\frac{2^{\mu+\nu-1} \Gamma(\nu) \Gamma((\mu+\nu+1) / 2)}{x^{\nu} \Gamma((1+\nu-\mu) / 2)} E\left(1-\nu ;-\frac{x^{2}}{4}\right) \\
& +\frac{2^{\mu-\nu-1} x^{\nu} \Gamma(-\nu) \Gamma((\mu-\nu+1) / 2)}{\Gamma((1-\nu-\mu) / 2)} E\left(1+v ;-\frac{x^{2}}{4}\right),
\end{aligned}
$$

where $(e)_{k}=e(e+1) \cdots(e+k-1)$ denotes the ascending factorial. We also need the following important lemma.

Lemma 1.1 (Prudnikov et al. [20, 21, equation (2.16.3.14), volume 2]). For $c>0, z>0$ and $v>-1$,

$$
\int_{0}^{\infty} \frac{x^{\nu+1}}{\left(x^{2}+z^{2}\right)^{\rho}} K_{\nu}(c x) d x=(2 z)^{\nu}(z / c)^{1-\rho} \Gamma(\nu+1) S_{-\nu-\rho, 1+\nu-\rho}(c z)
$$

Further properties of the above special functions can be found in Prudnikov et al. $[20,21]$ and Gradshteyn and Ryzhik [4].

\section{Cdf}

Theorem 2.1 derives an explicit expression for the cdf of $|X / Y|$ in terms of the Lommel function. 
Theorem 2.1. Suppose $X$ and $Y$ are distributed according to (1.2) and (1.3), respectively. If $a=2(M-1)$ is an odd integer then the $c d f$ of $Z=|X / Y|$ can be expressed as

$$
F(z)=I(a)+\frac{2 a^{m / 2} \Gamma(m+1)}{\pi^{3 / 2} b^{m+1} \Gamma(m+1 / 2) r^{m}} \sum_{k=1}^{(a-1) / 2} \frac{a^{k / 2} B(k, 1 / 2)}{b^{1-k} r^{k}} S_{-(m+k), 1+m-k}\left(\frac{\sqrt{a}}{b r}\right),
$$

where $I(\cdot)$ denotes the integral

$$
I(a)=\frac{1}{\pi^{3 / 2} 2^{m-2} b^{m+1} \Gamma(m+1 / 2)} \int_{0}^{\infty} \arctan \left(\frac{r y}{\sqrt{a}}\right) y^{m} K_{m}\left(\frac{y}{b}\right) d y,
$$

and $r=\sqrt{a / N} z$.

Proof. Using the relationship (1.5), one can write the cdf as $\operatorname{Pr}(|X / Y| \leq z)=\operatorname{Pr}(|U / Y| \leq$ $r$ ), which can be expressed as

$$
\begin{aligned}
F(r) & =\frac{1}{\sqrt{\pi} 2^{m} b^{m+1} \Gamma(m+1 / 2)} \int_{-\infty}^{\infty}\{F(r|y|)-F(-r|y|)\}|y|^{m} K_{m}\left(\left|\frac{y}{b}\right|\right) d y \\
& =\frac{1}{\sqrt{\pi} 2^{m-1} b^{m+1} \Gamma(m+1 / 2)} \int_{0}^{\infty}\{F(r y)-F(-r y)\} y^{m} K_{m}\left(\frac{y}{b}\right) d y,
\end{aligned}
$$

where $F(\cdot)$ inside the integrals denotes the cdf of a Student's $t$ random variable with degrees of freedom $a$. Substituting the form for $F$ given by (1.7) for odd degrees of freedom, (2.3) can be reduced to

$$
F(r)=I(a)+\frac{2 r}{\pi^{3 / 2} \sqrt{a} 2^{m} b^{m+1} \Gamma(m+1 / 2)} \sum_{k=1}^{(a-1) / 2} a^{k} r^{-2 k} B\left(k, \frac{1}{2}\right) J(k),
$$

where $J(k)$ denotes the integral

$$
J(k)=\int_{0}^{\infty} \frac{y^{m+1} K_{m}(y / b)}{\left(y^{2}+a / r^{2}\right)^{k}} d y
$$

By direct application of Lemma 1.1, one can calculate (2.5) as

$$
J(k)=2^{m} m ! a^{(m-k+1) / 2} b^{k-1} r^{k-m-1} S_{-(m+k), 1+m-k}\left(\frac{\sqrt{a}}{b r}\right) .
$$

The result of the theorem follows by substituting (2.6) into (2.4).

Theorem 2.2 is the analogue of Theorem 2.1 for the case when the degrees of freedom $2(M-1)$ is an even integer. 


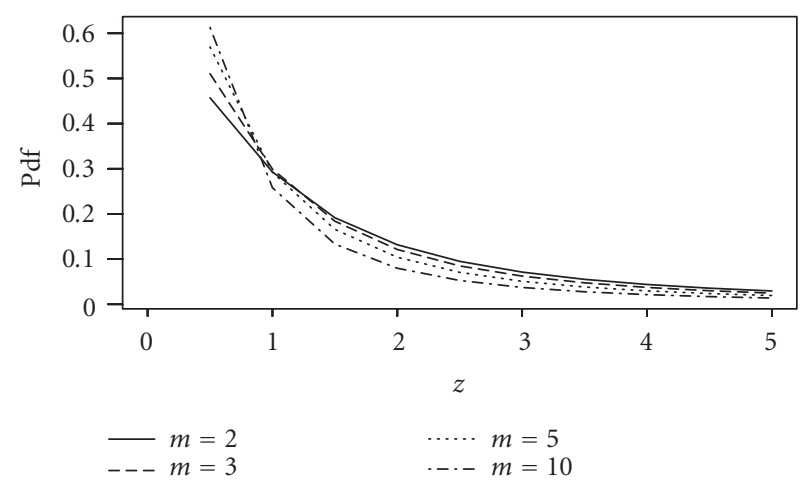

Figure 2.1. Plots of the pdf of (2.1) and (2.7) for $M=6, N=1$ and $m=2,3,5,10$.

Theorem 2.2. Suppose $X$ and $Y$ are distributed according to (1.2) and (1.3), respectively. If $a=2(M-1)$ is an even integer then the cdf of $Z=|X / Y|$ can be expressed as

$$
F(z)=\frac{2 a^{m / 2} \Gamma(m+1)}{\pi^{3 / 2} b^{m+1} \Gamma(m+1 / 2) r^{m}} \sum_{k=1}^{a / 2} \frac{a^{k / 2} B(k-1 / 2,1 / 2)}{b^{3 / 2-k} r^{k}} S_{1 / 2-m-k, 3 / 2+m-k}\left(\frac{\sqrt{a}}{b r}\right),
$$

where $r=\sqrt{a / N} z$.

Proof. Substituting the form for $F$ given by (1.7) for odd degrees of freedom, (2.3) can be reduced to

$$
F(r)=\frac{2 r}{\pi^{3 / 2} \sqrt{a} 2^{m} b^{m+1} \Gamma(m+1 / 2)} \sum_{k=1}^{a / 2} a^{k-1 / 2} r^{1-2 k} B\left(k-\frac{1}{2}, \frac{1}{2}\right) J(k),
$$

where $J(k)$ denotes the integral

$$
J(k)=\int_{0}^{\infty} \frac{y^{m+1} K_{m}(y / b)}{\left(y^{2}+a / r^{2}\right)^{k-1 / 2}} d y .
$$

By direct application of Lemma 1.1, one can calculate (2.9) as

$$
J(k)=2^{m} m ! a^{(m-k+3 / 2) / 2} b^{k-3 / 2} r^{k-m-3 / 2} S_{1 / 2-m-k, 3 / 2+m-k}\left(\frac{\sqrt{a}}{b r}\right) .
$$

The result of the theorem follows by substituting (2.10) into (2.8).

Figure 2.1 illustrates possible shapes of the pdf of $|X / Y|$ for $M=6, N=1$ and a range of values of $m$. Note that the shapes are unimodal and that the value of $m$ largely dictates the behavior of the pdf near $z=0$.

Table 2.1 provides percentage points of the random variable $Z=|X / Y|$, where $X$ is Student's $t$ random variable and $Y$ is the logistic random variable (see the next section). 
Table 2.1. Percentage points of $Z=|X / Y|$, where $X$ is Student's $t$ and $Y$ is logistic.

\begin{tabular}{|c|c|c|c|c|c|c|}
\hline$v$ & $p=0.9$ & $p=0.95$ & $p=0.975$ & $p=0.99$ & $p=0.995$ & $p=0.999$ \\
\hline 3 & 5.562765 & 11.38354 & 22.97968 & 58.01899 & 116.9898 & 590.5066 \\
\hline 4 & 6.262781 & 12.71831 & 25.40326 & 64.11429 & 126.6723 & 631.2136 \\
\hline 5 & 6.571752 & 13.28467 & 26.69873 & 66.59717 & 134.2463 & 683.6856 \\
\hline 6 & 6.713695 & 13.57901 & 27.17702 & 68.55331 & 137.8122 & 661.8825 \\
\hline 7 & 6.785755 & 13.71471 & 27.67064 & 69.98242 & 139.2899 & 706.187 \\
\hline 8 & 6.851048 & 13.84042 & 27.70334 & 69.84954 & 137.9891 & 718.6892 \\
\hline 9 & 6.890786 & 13.90781 & 27.73966 & 69.55903 & 137.6793 & 699.746 \\
\hline 10 & 6.896549 & 14.00395 & 28.24329 & 70.84583 & 142.5509 & 725.8325 \\
\hline 11 & 6.95357 & 14.02722 & 28.11031 & 69.5793 & 137.904 & 696.0801 \\
\hline 12 & 6.972723 & 14.09180 & 28.12042 & 69.88332 & 137.7253 & 672.2092 \\
\hline 13 & 6.98896 & 14.14476 & 28.17688 & 70.4751 & 141.4761 & 693.2014 \\
\hline 14 & 7.009541 & 14.11228 & 28.33336 & 71.51355 & 140.8694 & 705.5214 \\
\hline 15 & 7.037168 & 14.21723 & 28.54814 & 70.52729 & 140.4317 & 672.6095 \\
\hline 16 & 7.016891 & 14.15984 & 28.35549 & 71.11684 & 143.1923 & 749.5653 \\
\hline 17 & 7.036384 & 14.22758 & 28.58657 & 71.57068 & 141.4651 & 688.2397 \\
\hline 18 & 6.997855 & 14.16037 & 28.31962 & 70.2939 & 141.4549 & 705.8531 \\
\hline 19 & 7.013366 & 14.24295 & 28.55525 & 71.99197 & 144.5075 & 760.897 \\
\hline 20 & 7.05649 & 14.23309 & 28.37539 & 71.53259 & 142.1804 & 711.128 \\
\hline 21 & 7.079385 & 14.23683 & 28.82255 & 71.48645 & 140.3166 & 698.9362 \\
\hline 22 & 7.068098 & 14.29088 & 28.87514 & 71.84303 & 144.8472 & 720.8385 \\
\hline 23 & 7.065508 & 14.24519 & 28.45713 & 71.72072 & 142.1739 & 707.4103 \\
\hline 24 & 7.08719 & 14.26229 & 28.42829 & 71.54304 & 144.2094 & 744.5381 \\
\hline 25 & 7.088949 & 14.22109 & 28.56216 & 71.27478 & 141.8147 & 700.2782 \\
\hline 26 & 7.075956 & 14.22041 & 28.37632 & 69.83216 & 138.6226 & 714.1018 \\
\hline 27 & 7.061162 & 14.19190 & 28.37419 & 71.1976 & 140.5687 & 766.0238 \\
\hline 28 & 7.103002 & 14.28380 & 28.55252 & 72.33449 & 144.1237 & 715.3463 \\
\hline 29 & 7.087898 & 14.35654 & 28.80674 & 72.79021 & 144.8118 & 713.6059 \\
\hline 30 & 7.07424 & 14.2707 & 28.63380 & 71.4855 & 141.3878 & 698.7528 \\
\hline 31 & 7.089486 & 14.25152 & 28.65639 & 72.30395 & 145.2743 & 741.7811 \\
\hline 32 & 7.06058 & 14.19111 & 28.56882 & 72.30109 & 146.1088 & 691.8094 \\
\hline 33 & 7.137295 & 14.41838 & 28.77831 & 71.79008 & 143.2821 & 708.5934 \\
\hline 34 & 7.09389 & 14.28261 & 28.41822 & 70.6438 & 139.7121 & 716.6218 \\
\hline 35 & 7.085203 & 14.25356 & 28.53685 & 71.72688 & 143.7028 & 682.4963 \\
\hline 36 & 7.109764 & 14.32743 & 28.82298 & 71.98 & 143.0642 & 729.9266 \\
\hline 37 & 7.103148 & 14.32635 & 28.89781 & 72.73917 & 144.9194 & 720.3259 \\
\hline 38 & 7.119519 & 14.37951 & 29.07110 & 72.8409 & 147.2216 & 729.187 \\
\hline 39 & 7.099915 & 14.36532 & 28.65924 & 71.41699 & 144.1128 & 748.3013 \\
\hline 40 & 7.097136 & 14.24074 & 28.39764 & 70.28753 & 139.6711 & 709.3462 \\
\hline 41 & 7.121472 & 14.36992 & 28.91728 & 71.5884 & 145.9546 & 687.5026 \\
\hline 42 & 7.122682 & 14.34184 & 28.83802 & 72.30898 & 143.8715 & 708.7313 \\
\hline
\end{tabular}


Table 2.1. Continued.

\begin{tabular}{lclllll}
\hline$\nu$ & $p=0.9$ & $p=0.95$ & $p=0.975$ & $p=0.99$ & $p=0.995$ & $p=0.999$ \\
\hline 43 & 7.136523 & 14.31962 & 28.82072 & 72.56791 & 145.7568 & 726.197 \\
44 & 7.073623 & 14.31739 & 28.59732 & 70.96285 & 143.9753 & 684.8288 \\
45 & 7.098625 & 14.29619 & 28.63336 & 70.971 & 141.1438 & 771.8231 \\
46 & 7.076823 & 14.29069 & 28.68125 & 71.09681 & 141.0267 & 691.0396 \\
47 & 7.133042 & 14.37075 & 28.72546 & 72.23762 & 144.6101 & 741.7482 \\
48 & 7.111953 & 14.33463 & 28.78489 & 71.15746 & 141.5647 & 746.4949 \\
49 & 7.109182 & 14.25147 & 28.80318 & 72.11692 & 142.8293 & 700.7086 \\
50 & 7.119438 & 14.31630 & 28.71464 & 72.46818 & 144.9791 & 702.7882 \\
\hline
\end{tabular}

\section{Application}

In this section, we provide "correction factors" for the approximation (1.1). These factors are computed as the percentage points $z_{p}$ associated with the cdfs (2.1) and (2.7) when $X$ is a Student's $t$ random variable with degrees of freedom $v$ and $Y$ is a logistic random variable with the scale parameter $\pi \sqrt{(\nu-2) /(3 v)}$. Evidently, this involves computation of the Lommel and Bessel functions. Fortunately routines for these calculations are widely available. We used the functions LommelS $1(\cdot)$, LommelS $2(\cdot)$, and BesselK $(\cdot)$ in the algebraic manipulation package, MAPLE. Table 2.1 provides the numerical values of $z_{p}$ for $v=3,4, \ldots, 50$ and $p=0.9,0.95,0.975,0.99,0.995,0.999$. We hope these numbers will be of use to the practitioners of the approximation (1.1). Similar tabulations could be easily derived for other values of $\nu$ by using the LommelS $1(\cdot)$, LommelS2(*), and $\operatorname{BesselK}(\cdot)$ functions in MAPLE.

\section{Acknowledgments}

The authors would like to thank the referee and the editor for carefully reading the paper and for their assistance in improving the paper.

\section{References}

[1] A. P. Basu and R. H. Lochner, On the distribution of the ratio of two random variables having generalized life distributions, Technometrics 13 (1971), 281-287.

[2] E. O. George, M. El-Saidi, and K. Singh, A generalized logistic approximation of the Student $t$ distribution, Comm. Statist. B-Simulation Comput. 15 (1986), no. 4, 1199-1208.

[3] E. O. George and M. O. Ojo, On a generalization of the logistic distribution, Ann. Inst. Statist. Math. 32 (1980), no. 2, 161-169.

[4] I. S. Gradshteyn and I. M. Ryzhik, Table of Integrals, Series, and Products, 6th ed., Academic Press, California, 2000.

[5] D. L. Hawkins and C.-P. Han, Bivariate distributions of some ratios of independent noncentral chi-square random variables, Comm. Statist. A-Theory Methods 15 (1986), no. 1, 261277.

[6] D. V. Hinkley, On the ratio of two correlated normal random variables, Biometrika 56 (1969), 635-639. 
[7] N. L. Johnson and S. Kotz, Distributions in Statistics: Continuous Univariate Distributions. 2, 1st ed., Houghton Mifflin, Massachusetts, 1970.

[8] R. F. Kappenman, A note on the multivariate t ratio distribution, Ann. Math. Statist. 42 (1971), 349-351.

[9] P. J. Korhonen and S. C. Narula, The probability distribution of the ratio of the absolute values of two normal variables, J. Statist. Comput. Simulation 33 (1989), no. 3, 173-182.

[10] S. Kotz, T. J. Kozubowski, and K. Podgórski, The Laplace Distribution and Generalizations: A Revisit with Applications to Communications, Economics, Engineering, and Finance, Birkhäuser Boston, Massachusetts, 2001.

[11] S. Kotz and S. Nadarajah, Multivariate t Distributions and Their Applications, Cambridge University Press, Cambridge, 2004.

[12] R. Y. Lee, B. S. Holland, and J. A. Flueck, Distribution of a ratio of correlated gamma random variables, SIAM J. Appl. Math. 36 (1979), no. 2, 304-320.

[13] B. Li and B. De Moor, A simple approximation relation between the symmetric generalized logistic and the Student's t distributions, Comm. Statist. Simulation Comput. 26 (1997), no. 3, 10291039.

[14] G. Marsaglia, Ratios of normal variables and ratios of sums of uniform variables, J. Amer. Statist. Assoc. 60 (1965), 193-204.

[15] G. S. Mudholkar and E. O. George, A remark on the shape of the logistic distribution, Biometrika 65 (1978), 667-668.

[16] S. Nadarajah and S. Kotz, Skewed distributions generated by the normal kernel, Statist. Probab. Lett. 65 (2003), no. 3, 269-277.

[17] T. Pham-Gia, Distributions of the ratios of independent beta variables and applications, Comm. Statist. Theory Methods 29 (2000), no. 12, 2693-2715.

[18] S. J. Press, The t-ratio distribution, J. Amer. Statist. Assoc. 64 (1969), 242-252.

[19] S. B. Provost, On the distribution of the ratio of powers of sums of gamma random variables, Pakistan J. Statist. 5 (1989), no. 2, 157-174.

[20] A. P. Prudnikov, Y. A. Brychkov, and O. I. Marichev, Integrals and Series. Vol. 1. Elementary Functions, Gordon \& Breach Science, New York, 1986.

[21] - Integrals and Series. Vol. 2. Special Functions, Gordon \& Breach Science, New York, 1986.

[22] , Integrals and Series. Vol. 3. More Special Functions, Gordon \& Breach Science, New York, 1990.

[23] S. M. Shcolnick, On the ratio of independent stable random variables, Stability Problems for Stochastic Models (Uzhgorod, 1984), Lecture Notes in Math., vol. 1155, Springer, Berlin, 1985, pp. 349-354.

Saralees Nadarajah: Department of Statistics, University of Nebraska, Lincoln, NE 68583, USA

E-mail address: snadaraj@unlserve.unl.edu

Samuel Kotz: Department of Engineering Management and Systems Engineering, George Washington University, Washington, DC 20052, USA

E-mail address: kotz@gwu.edu 


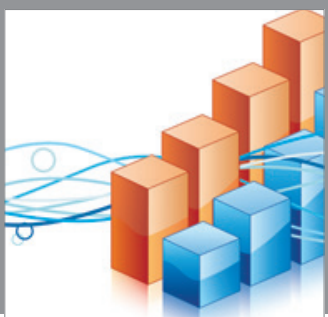

Advances in

Operations Research

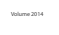

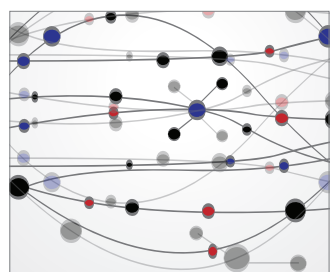

\section{The Scientific} World Journal
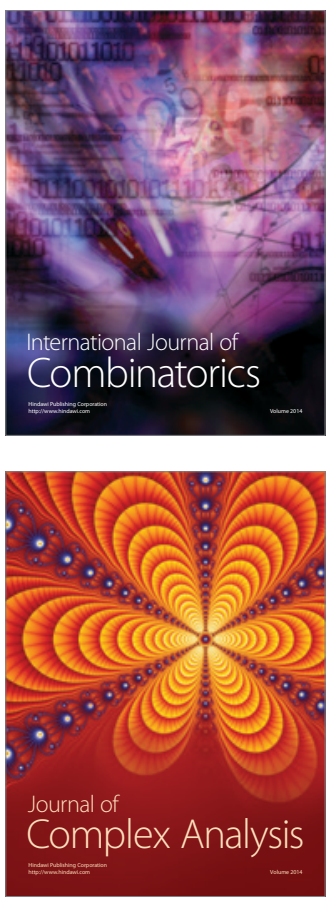

International Journal of

Mathematics and

Mathematical

Sciences
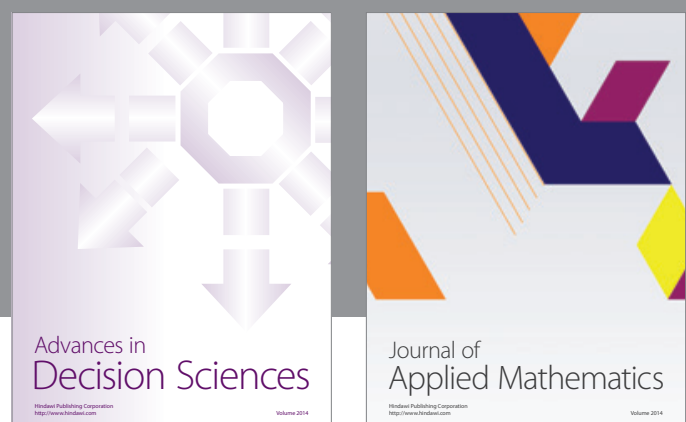

Journal of

Applied Mathematics
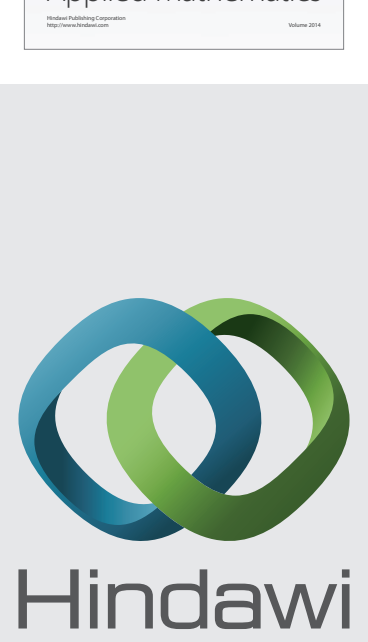

Submit your manuscripts at http://www.hindawi.com
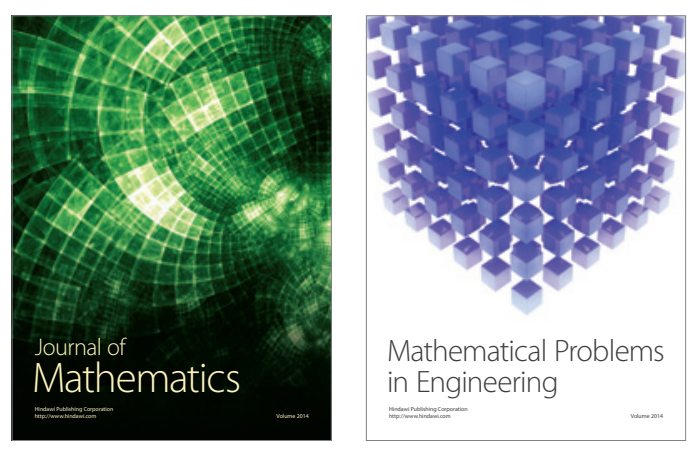

Mathematical Problems in Engineering
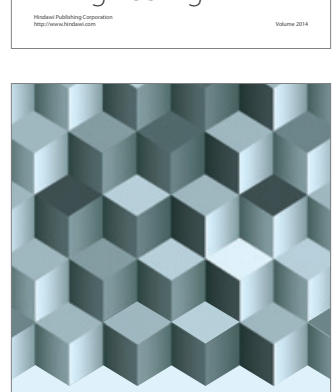

Journal of

Function Spaces
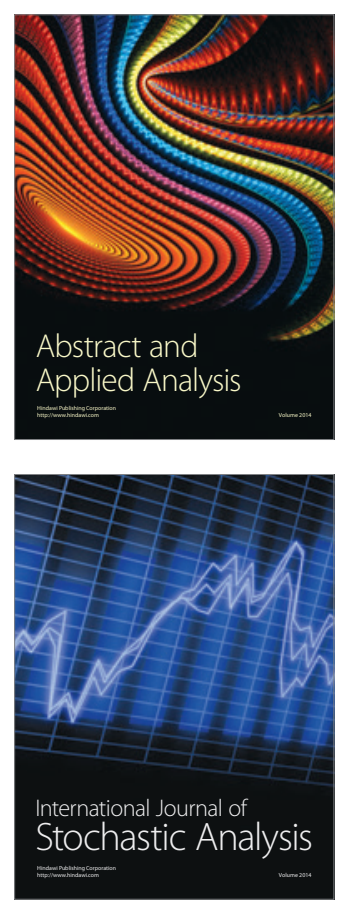

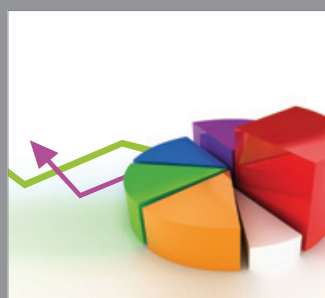

ournal of

Probability and Statistics

Promensencen
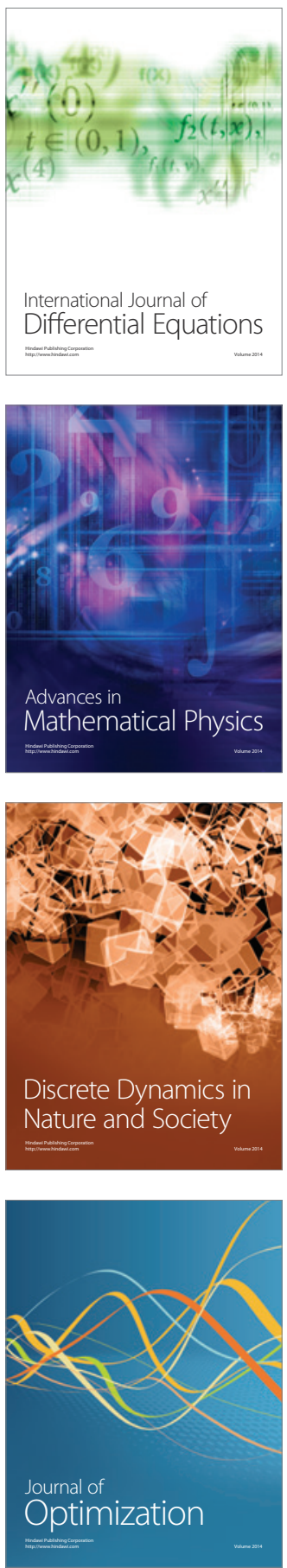